\title{
Artificial intelligence in medicine and dermatology
}

\author{
Anna Woźniackaํ, Sebastian Patrzyk ${ }^{1}$, Maksym Mikołajczyk ${ }^{2}$ \\ ${ }^{1}$ Department of Dermatology and Venereology, Medical University of Lodz, Lodz, Poland \\ ${ }^{2}$ Student Research Circle at the Department of Dermatology and Venereology, Medical University of Lodz, Lodz, Poland \\ Adv Dermatol Allergol 2021; XXXVIII (6): 948-952 \\ DOI: https://doi.org/10.5114/ada.2020.101259
}

\begin{abstract}
As technology advances rapidly and vast amounts of data are collected, artificial intelligence (Al) is increasing its presence in our lives. Medicine is a major focus point of Al developers. There are examples of algorithms on par with medical professionals, the most prominent case being skin cancer recognition. However, advancement involves the necessity to adapt to technology and to patients utilizing it on a daily basis. What is more, patients present growing trust towards machine-aided health care. Dermatology is a potent field for Al use as visual data are easy to collect, hold a lot of information and are paramount for diagnosis.
\end{abstract}

Key words: artificial intelligence, new technology, education.

\section{Introduction}

Medicine has undergone a rapid development over the past 20 years. Medical professionals now have to cope with new diseases and new understandings of old diseases as well as new medications and diagnostic methods. The professional environment in which we work in is constantly changing and for medicine to evolve, it must adapt to it.

Not so long ago, only physicians were privileged to have access to medical information, and with this access, they were duty bound to educate patients about general health and disease. As the primary source of medical education, doctors were able to provide relevant and proper medical advice. Nowadays, the patient enters the doctor's office armed with information received from wearables, Doctor Google, mobile applications, social media and chat bots. Unfortunately, most of this information is of questionable quality and it is uncertain whether such unrestricted access is beneficial to the patient. Nevertheless, physicians must be prepared for such interactions and develop strategies for working with such new patients and new technologies as both opportunities and challenges lie ahead.

Artificial intelligence (Al), sometimes called machine intelligence, is the term given to a software system that simulates human intelligence processes by using data inputs to make independent decisions or help users make decisions. However, at present many different defini- tions of Al exist. It has been defined as a system with the ability to correctly interpret external data, to learn from such data, and to use those inferences to achieve specific goals and tasks through flexible adaptation [1]. Colloquially, the term $\mathrm{Al}$ is applied when machine mimics cognitive functions that humans associate with other human minds, such as learning and problem solving [2].

Al systems and rapid advancements in digital technologies are revolutionizing healthcare. They have the potential to establish the diagnosis and introduce treatment of diseases and play a crucial role in many learning processes [3].

In healthcare, Al is often applied for the large-scale analysis of big data associated with health information, such as patient age, medical history, health status, test results, medical images, and DNA sequences. As Al can work faster than human researchers and without breaks, it can clearly offer a number of advantages for improving healthcare. The question arises about how it can be integrated successfully into the healthcare systems. The first step is to be familiar with electronic systems and know what Al truly means.

When discussing the use of such technology in medicine, it is important to make a distinction between Al and augmented intelligence (Aul), which combines human and machine intelligence and is aimed at supporting human intelligence rather than replacing it. Al systems can run without humans, whereas Aul aims to create sys-

Address for correspondence: Maksym Mikołajczyk, Department of Dermatology and Venereology, Medical University of Lodz, pl. Hallera 1, 90-647 Lodz, Poland, phone: +48 42 6393092, fax: +48 42 6884565, e-mail: maksym.m@onet.eu Received: 23.01.2020, accepted: 8.02.2020. 
tems that make humans better. There has recently been a growth in interest in the use of Aul in dermatology, with various mobile applications being developed to support diagnoses and medical decisions. Over the next 20 years, Aul will doubtlessly play a role in establishing diagnoses, introducing treatments and identifying places of referral.

\section{Diagnosis}

The diagnostic process comprises a medical history and a physical examination. In dermatology, the analysis of skin images is a fundamental part of the latter, and a thorough knowledge of different types of skin lesions, their distribution and symmetry is essential. Many procedures, such as histopathological or immunofluorescence evaluation, dermoscopy and ultrasonography, are based on digital image analysis. In contrast, the medical history is typically collected through an interview with the patient, and comprises not only a general history of the particular skin disease, but also the occupation, hobbies, routine household activities, animal contact and diet of the patient, as well as various other miscellaneous factors such as seasonal variation of the disease, association with menses or pregnancy, concomitant disorders, medication history and family history.

The growth in processing power of computers has been matched by the growth of more complex sources of data. It is now possible to electronically access an entire health record, including data pulled from daily life by wearable devices or smartphones: this can include such everyday activities as the number of steps walked, sleep patterns, hypertension, pulse rates, nutrition record or even blood saturation.

The most powerful tool for acquiring such data is undoubtedly the smartphone: it is always available, and can be used to measure a range of aspects of the health status. Being equipped with a camera, motion sensors, accelerometer, fast processing power and access to unlimited internet data and applications it is an ideal healthcare assistant. Unfortunately, some smartphone applications may be inaccurate, leading to false negative diagnoses of malignant changes. Most applications have not undergone published trials to show that they work correctly and are safe; they also lack validation and scientific input from specialists during development and the technology itself may be subject to errors [4].

There is a general agreement that new technologies can help doctors and patients make better and faster healthcare decisions. Currently, the ability to form a correctly diagnosis takes years of medical training, and even then, diagnostics is often a difficult and time-consuming process. Huge advances in automatically diagnosing of diseases have recently been made by Machine Learning, particularly deep learning algorithms. Machine learning algorithms can learn to see patterns similarly to the way doctors see them; however, a key difference is that these algorithms need a lot of concrete examples, usually many thousands to make accurate predictions [5]. In addition, they require the diagnostic information to be digitized: lung cancer or strokes can be diagnosed based on computed tomography (CT) scans, the risk of sudden cardiac death or other heart diseases can be evaluated by electrocardiograms and cardiac magnetic resonance imaging (MRI) images, skin lesions categorized by skin images and diabetic retinopathy identified by eye images.

Al systems for health care have an enormous potential to transform the diagnosis and treatment of diseases. However, there are many challenges for implementing this new technology in medical practice. Al requires strong processing power and large databases; in addition, as data are the fuel of computer learning, such data must be of high quality. Methods of evaluating whether an Al system is actually better than standard medical care need to be developed. Importantly, patient privacy must be protected. Systems that analyse data in health care are not the same as those that manage logistics for online orders: there is a need to extend the Hippocratic oath and adopt a proper, moral and ethical obligation to protect and use patient data appropriately. Perhaps the greatest criticism of the use of such machine-based technology is that it is widely felt that machines can never replace the emotions, creativity, empathy, sense of humour and basic intuition which are required for medical professionals to engage with patients.

Years of medical training are required to develop the ability to correctly diagnose skin disorders, which itself is a time-consuming process. Machine learning, particularly the use of deep learning algorithms, has enabled automatic identification, making diagnostics cheaper and faster; these diagnoses are sometimes also more accurate than those of a general practitioner or even a specialist [5].

It is possible for Al to detect subtle changes in mammograms, early patterns of cancer in X-ray or CT scans, as well as early patterns of heart failure or typical patterns of skin nevi and skin lesions that may be missed by a highly-trained physician.

However, dermatological Al faces a number of problems that must be addressed before its wide-scale implementation. Firstly, the current database of skin disease images is still unsatisfactory, with a lack of labelled data and samples suitable for the complex network model. Secondly, the combination of medical and Al complex abilities is hampered by a lack of cooperation between multi-disciplinary workers in computer science and biomedicine, as well as medical health care professionals. Thirdly, Al can recognize only a few out of many kinds of specific skin disorders. Fourthly, the current Al diagnosis comprises numerous legal and ethical issues [6]. As mentioned above, the identification of skin diseases involves not only an analysis of skin images, but also a range of clinical tests and a patient history; therefore, skin image 
data and patient information need to be combined to allow systematic evaluation by Al [7]. As yet, no dermatological Al product has yet obtained formal recognition by a professional body.

\section{Therapy}

Digital therapeutics are defined as any intervention that is digitally delivered and has a therapeutic effect on a patient. Many applications are currently available for managing medications, maintaining cardiovascular health, and supporting mental health and well-being. The term connected health has been coined to reflect the environment in which clinicians and patients increasingly communicate through digital media such as text messaging or video consultations with health care professionals.

The US health care system, and those of many European countries, are characterized by long waiting times. Moreover, due to the small number of consultants, in cluding practicing dermatologists, diagnoses can be expensive. In such cases, health care costs and waiting times can be reduced, and medical outcomes improved, by eliminating unnecessary visits; one such approach would be to set a general diagnosis automatically and then refer the patient to a doctor for a consultation. In addition, there is increasing evidence that digitally delivered care, such as smartphone apps and text messagebased interventions, can help to manage chronic conditions such as psoriasis, chronic leg ulcers, diabetes or cardiovascular diseases.

Medical strategies, including digital health devices, have the potential to play a unique and important part of the treatment process. In 2018, the Food and Drug Administration approved a mobile medical application for treating opioid use disorder (OUD). reSET-O is an application that can be downloaded directly to a mobile device on prescription. This application can serve as a training, monitoring and reminder tool for both health care providers and patients in maintaining an outpatient treatment program. Patients who used the desktop computer version of the reSET-O program demonstrated significantly greater retention on a 12 -week treatment program (82.4\%) than those who did not (68.4\%). The application is not intended to be used as a stand-alone therapy, a substitute for medication, or for patients whose primary language is not English [8].

Recent studies have focused on the design of more complicated systems involving the combination of multiple data sources like photographs, Computer Tomography, Magnetic Resonance, genomics and proteomics, patient data, and even recorded speech or handwritten files in assessing a disease or its progression. What is more, machine learning can identify the characteristics indicating that a patient will have a particular response to a specific treatment.
One computer system prepared for the health care sector as a 'question answering machine' is WATSON, named after IBM's founder Thomas J. Watson. It combines $\mathrm{Al}$ and sophisticated analytical software and offers enormous potential advantages over human doctors. It possesses information about every known illness and medicine, and can be very quickly familiarized with the symptoms, disease medical history and lifestyle of the patient, as well as genetic information. It is currently easier to replace physicians with a narrow specialization than can be easy digitized, particularly areas that employ data that could be reduced to algorithms, such as CT scans, mammography and skin lesion images [9].

In conjunction with Al demonstrating humanlike interaction abilities, such as the Google Assistant (Google LLC, Mountain View, CA; most prominent example of live interaction: https://www.youtube.com/ watch?v=pKVppdt_-B4), such a system could conduct automated patient interviews in places like emergency departments, where time is crucial and filtering out important symptoms allows patient care prioritization.

Babylon is the first service of its kind to be registered with the Care Quality Commission, the British health care services regulator [10] is a subscription health service provider that offers a digital health care application using a mixture of Al and video and text consultations with doctors and specialists. The service also allows users to receive drug prescriptions. The company also claims that its Al has the ability to diagnose health issues better than certified physicians in the United Kingdom [11].

A study of patient acceptance and trust in medical technology found that while most participants believed that a computer could improve the performance of a physician, almost all preferred the expert physician opinion besides the computer-assisted diagnosis [12].

\section{Education}

Paper-based records, prescription charts and education publications have been replaced by emails, online forms, electronic databases, open access journals and electronic medication. Al may well improve the education of both patients and care providers through the use of augmented and virtual reality tools.

With help from Al, education can be personalized to students of different ages, level of prior skill, learning style and preference or even cognitive prowess. Vast resources could be searched with state-of-the-art algorithms to identify specific information. They could also be converted into easy-to-use flashcards. Al could also help skip the cumbersome procedure of processing raw textbook data into notes [13].

Moreover, quality learning is a twofold process comprising internalizing knowledge and its external evaluation. New technologies can also offer opportunities for self-directed learning. For example, Al can act as a part- 
ner for language practice [14]. There is probably a long way to go for such applications teaching tone languages, like Chinese or Vietnamese, but can work perfectly for Indo-European languages.

Feedback can also be achieved with the use of smart chat bots that provide greater efficiency of learning and student satisfaction [15]. If Al can process and summarize a complex problem and explain it in simple words, interactively answering questions, the student can progress without discouragement.

Intelligent Tutoring Systems (ITS) use Al techniques to adapt tutoring strategies to learners according to what they know about the target domain [16]. They are designed to provide learners with instant and personalized instructions or feedback. One such system is SlideTutor, an ITS aimed at tutoring microscopic diagnosis of inflammatory skin diseases [17].

Virtual Reality (VR) can also be used to train medical staff in safe conditions without involving patients. These applications can be greatly enhanced through the use of Al or Aul to determine the position of objects, such as hands and fingers, within the simulation. Such applications are used in a range of disciplines such as endoscopic surgery [18], echocardiography [19], neurosurgery [20] and orthopaedics [21]. The TutorDerm system is one such VR application intended for dermatology which enables the simulation of dermatological procedures [22].

\section{Conclusions}

Although the influence of $\mathrm{Al}$ on medicine is growing, it is important to remember that the software is intended to be a tool to support the physician, and that it is the physician who is ultimately responsible for diagnosis and treatment. There is little chance of Al replacing physicians in the near future; however, medical professionals who use Al will replace those who do not. Our future rests in partnership with intelligent machines and algorithms.

\section{Acknowledments}

This work was supported by the grant from the Medical University of Lodz, number 503/1-152-01/503-11-001-19-00.

Sebastian Patrzyk and Maksym Mikołajczyk - equal contribution.

\section{Conflict of interest}

The authors declare no conflict of interest.

\section{References}

1. Andreas K, Haenlein M. Siri, Siri in my hand, who's the Fairest in the Land? On the Interpretations, Illustrations and Implications of Artificial Intelligence. Business Horizons 2019; 62: $15-25$.
2. Russel S, Norvig P. Artificial Intelligence: a Modern Approach. $3^{\text {rd }}$ edition. Prentice Hall; Upper Saddle River, New Jersey 2009.

3. Maddox TM, Rumsfeld JS, Payne PR. Questions for artificial intelligence in health care. JAMA 2019; 32: 31-2.

4. Charalambides M. Dermatology skin cancer applications the future of healthcare provision. I Natl Student Assoc Med Res 2018; 1: 45-9.

5. Esteva A, Kuprel B, Novoa RA, et al. Dermatologist level classification of skin cancer with deep neural networks. Nature 2017; 542: 115-8.

6. Li CX, Shen CB, Xue K, et al. Artificial intelligence in dermatology: past, present, and future. Chin Med J 2019; 132: 2017-20.

7. Haenssle HA, Fink C, Schneiderbauer R, et al. Man against machine: diagnostic performance of a deep learning convolutional neural network for dermoscopic melanoma recognition in comparison to 58 dermatologists. Ann Oncol 2018; 29: 1836-42.

8. Christensen DR, Landes RD, Jackson L, et al. Adding an Internet-delivered treatment to an efficacious treatment package for opioid dependence. J Consult Clin Psychol 2014; 82: 964-72.

9. Davenport T, Kalakota R. The potential for artificial intelligence in healthcare. Future Healthc J 2019; 6: 94-8.

10. O'Hear, S. Babylon Health raises further $\$ 60 \mathrm{M}$ to continue building out Al doctor app. [online] TechCrunch 2017. Available at: https://techcrunch.com/2017/04/25/babylonhealth-raises-further-60m-to-continue-building-out-ai-doctor-app/ [Accessed 21 Dec. 2019].

11. Bresnick J. Arguing the Pros and Cons of Artificial Intelligence in Healthcare. [online] Health IT Analytics 2018. Available at: https://healthitanalytics.com/news/arguingthe-pros-and-cons-of-artificial-intelligence-in-healthcare [Accessed 21 Dec. 2019].

12. Fink C, Uhlmann L, Hofmann M, et al. Patients acceptance and trust in automated computer-assisted diagnosis of melanoma with dermatofluoroscopy. J Dtsch Dermatol Ges 2018; 16: 854-9.

13. Schmelzer R. Al Applications In Education. [online] Forbes. Available at: https://www.forbes.com/sites/cognitiveworld/2019/07/12/ai-applications-in-education/ [Accessed 21 Dec. 2019].

14. Petsko E. Language Learning App Magiclingua Lets You Practice Speaking With an Al-Powered Smart Bot. [online] Mentalfloss. Available at: https://www.mentalfloss.com/article/581777/magiclingua-ai-powered-language-learning-app [Accessed 21 Dec. 2019].

15. Faggella D. Examples of Artificial Intelligence in Education|Emerj. [online] Emerj. Available at: https://emerj.com/ai-sector-overviews/examples-of-artificial-intelligence-in-education/ [Accessed 21 Dec. 2019].

16. Sleeman D. Brown JS, Intelligent Tutoring Systems. Academic Pres, London 1982.

17. Crowley RS, Medvedeva O, Jukic D. Slide tutor: a modeltracing intelligent tutoring system for teaching microscopic diagnosis. Artificial Intelligence in Education 2003; 157-64.

18. Gurusamy K, Aggarwal R, Palanivelu L, et al. Systematic review of randomized controlled trials on the effectiveness of virtual reality training for laparoscopic surgery. Br J Surg 2008; 95: 1088-97.

19. van den Bosch AE, Koning AH, Meijboom FJ, et al. Dynamic $3 \mathrm{D}$ echocardiography in virtual reality. Cardiovasc Ultrasound 2005; 3: 37. 
20. Alaraj A, Lemole MG, Finkle JH, et al. Virtual reality training in neurosurgery: review of current status and future applications. Surg Neurol Int 2011; 2: 52.

21. Vankipurama M. Kaholab K. McLarenc A. Et al. A virtual reality simulator for orthopedic basic skills: a design and validation study. J Biomed Informatics 2010; 43: 661-8.

22. Invention Med. TutorDerm Project. [online] Available at: https://inventionmed.com/projects/tutorderm-project/ [Accessed 21 Dec. 2019]. 